\title{
LINEAR AND NONLINEAR BOUNDARY CROSSING PROBABILITIES FOR BROWNIAN MOTION AND RELATED PROCESSES
}

\author{
JAMES C. FU* AND \\ TUNG-LUNG WU, ${ }^{* *}$ University of Manitoba
}

\begin{abstract}
We propose a new method to obtain the boundary crossing probabilities or the first passage time distribution for linear and nonlinear boundaries for Brownian motion. The method also covers certain classes of stochastic processes associated with Brownian motion. The basic idea of the method is based on being able to construct a finite Markov chain, and the boundary crossing probability of Brownian motion is cast as the limiting probability of the finite Markov chain entering a set of absorbing states induced by the boundaries. Error bounds are obtained. Numerical results for various types of boundary studied in the literature are provided in order to illustrate our method.

Keywords: Boundary crossing probability; first passage time; Brownian motion; diffusion process; finite Markov chain imbedding; discretization; random walk

2010 Mathematics Subject Classification: Primary 60J65

Secondary 60J70; 60J60; 60J10
\end{abstract}

\section{Introduction}

Brownian motion is one of the most important continuous-time stochastic processes, and boundary crossing probabilities (BCPs) or first passage times of one-dimensional Brownian motion type processes have wide applications in many fields, including nonparametric statistics (see [8] and [24]), sequential analysis (see [1] and [24]), mathematical finance (see [19]), biology (see [20] and [21]), change-point problems (see [24]), and many engineering problems.

Let $\{W(t): t \in[0, \infty)\}$ be a stochastic process defined on $\mathbb{R}$. The boundary crossing probability of $W(t)$ is

$$
\mathrm{P}(a(t) \geq W(t) \text { or } W(t) \geq b(t) \text { for some } t \in[0, T]),
$$

where $T$ is fixed, and $a(t)$ and $b(t)$ are continuous functions on [0,T]. There are various definitions for a Brownian motion. For our approach, we view the Brownian motion $W(t)$ as a Markov process normally distributed with mean 0 and variance $t$, independent increments, and continuous at 0 . Given $t>0$, the Brownian motion has density function

$$
f(x, t)=\frac{1}{\sqrt{2 \pi t}} \exp \left(\frac{x^{2}}{2 t}\right),
$$

which satisfies the differential equation

$$
\frac{\partial}{\partial t} f(x, t)=\frac{1}{2} \frac{\partial^{2}}{\partial x^{2}} f(x, t) .
$$

Received 20 October 2009; revision received 6 August 2010.

* Postal address: Department of Statistics, University of Manitoba, Winnipeg, MB, R3T 2N2, Canada.

** Email address: umwu48@cc.umanitoba.ca 
There are several historical and recent results for boundary crossing probabilities of Brownian motion. We briefly introduce a few in the following. Given $c>0$ and $\mathrm{P}(W(0)=0)=1$ (this condition is assumed throughout this paper), it follows from the reflection principle that the boundary crossing probability in the time interval $t \in[0, T]$ is

$$
\mathrm{P}\left(\sup _{0 \leq t \leq T} W(t) \geq c\right)=2 \mathrm{P}(W(T)>c)=\frac{2}{\sqrt{2 \pi T}} \int_{c}^{\infty} \exp \left(\frac{-x^{2}}{2 T}\right) \mathrm{d} x .
$$

For a one-sided boundary $b(t)$, a well-known tangent approximation was first introduced by Strassen [25] and independently established by Daniels [7] for general $t$ with the method of images. For $-\infty<a<0<b<\infty$, the two-sided boundary crossing probability for $t \in[0, T]$ was given in an infinite series form in [1]. For a linear boundary $a+b t, a>0$, Robbins and Siegmund [18] showed, using martingale theory, that

$$
\mathrm{P}\left(\sup _{0 \leq t \leq T}(W(t)-b t) \geq a\right)=1-\Phi\left(\frac{a}{\sqrt{T}}+b \sqrt{T}\right)+\exp (-2 a b) \Phi\left(b \sqrt{T}-\frac{a}{\sqrt{T}}\right),
$$

where $\Phi$ stands for the cumulative distribution of a standard normal distribution. Scheike [23] extended Robbins and Siegmund's [18] result to a piecewise linear boundary. Also, a number of papers obtained the boundary crossing probability as an integral equation or in integral form. Durbin [8], [9] computed the boundary crossing probabilities using the numerical solution of integral equations. Wang and Pötzelberger [26] and Pötzelberger and Wang [17] extended the results of Robbins and Siegmund [18] and Scheike [23] to approximate the boundary crossing probabilities for one-sided and two-sided nonlinear boundaries. Several general nonlinear boundaries have been considered and numerical computations were done using Monte Carlo simulation methods. Novikov et al. [16] also obtained bounds by piecewise approximation for two-sided boundary crossing probabilities. More approximations and computational algorithms for boundary crossing probabilities can be found in [22].

General diffusion processes governed by stochastic differential equations are often used as models in finance or biology (see, e.g. [20]). One of the methods used to find the boundary crossing probabilities for diffusion processes is to express the diffusion processes in terms of functions of the Brownian motion, and then the boundary crossing probabilities for diffusion processes can be obtained via the boundary crossing probabilities for Brownian motion with transformed time intervals and boundaries.

It is well known that Brownian motion can be approximated by discrete random walks moving by \pm 1 with equal probability (see, e.g. [11]). In this paper, a new approach inspired by the above idea is provided to obtain the boundary crossing probabilities of Brownian motion for nonlinear boundaries, using the strong Markov property of Brownian motion and the finite Markov chain imbedding technique (see [10]). It follows that the boundary crossing probability can be expressed in terms of transition matrices of a discrete Markov chain, i.e.

$$
\begin{aligned}
& \mathrm{P}(W(t) \leq a(t) \text { or } W(t) \geq b(t) \text { for some } t \in[0, T]) \\
& \quad=1-\mathrm{P}(a(t)<W(t)<b(t) \text { for all } t \in[0, T]) \\
& \quad=1-\lim _{n \rightarrow \infty} \xi_{0}\left(\prod_{i=1}^{n} N_{i}\right) \mathbf{1}^{\top},
\end{aligned}
$$

where $\mathbf{1}^{\top}$ is the transpose of the row vector $\mathbf{1}=(1, \ldots, 1)$, and the $N_{i}, i=1, \ldots, n$, are referred to as fundamental transient probability matrices of a discrete Markov chain $\left\{Y_{n}\right\}$ having absorbing states induced by boundaries $a(t)$ and $b(t)$. 
This paper is organized as follows. In Section 2 we provide some results for discrete-time Markov chains with finite state space. In Section 3 we provide the finite Markov imbedding procedure and main theorems on the boundary crossing probability for Brownian motion. In Section 4 we study the error bound. In Section 5 we provide several numerical results for linear and nonlinear boundary crossing probabilities to illustrate our theoretical results. A summary and discussion are given in Section 6.

\section{Finite Markov chain with absorbing state}

To facilitate our approach, we need a simple result for computing the absorption probability of a finite nonhomogeneous Markov chain. Given $n \in J^{+}=\{1,2, \ldots\}$, let us define a sequence of state spaces

$$
\Omega_{i}=\left\{c_{1}, c_{2}, \ldots, c_{m_{i}}\right\} \cup\left\{\alpha_{i}\right\} \text { for } i=1,2, \ldots, n,
$$

where $\alpha_{i}$ stands for an absorbing state and $m_{i}+1$ is the size of state space $\Omega_{i}$ (the $m_{i}$ do not have to be the same). Assume that $\Omega_{0}=\left\{c_{0}\right\}$, and denote by $\xi_{0}$ the initial distribution. Furthermore, assume that a finite Markov chain $\left\{Y_{i}\right\}_{i=0}^{n}$ is defined on the sequence of state spaces $\left\{\Omega_{i}\right\}_{i=0}^{n}$ with transition probabilities given by, for $i=1,2, \ldots, n$,

$$
\mathrm{P}\left(Y_{i}=k \mid Y_{i-1}=j\right)= \begin{cases}p_{i}(k \mid j) & \text { if } j \in \Omega_{i-1} \backslash \alpha_{i-1}, k \in \Omega_{i} \backslash \alpha_{i} \\ p_{i}\left(\alpha_{i} \mid j\right) & \text { if } j \in \Omega_{i-1} \backslash \alpha_{i-1} \\ 0 & \text { if } j=\alpha_{i-1}, k \in \Omega_{i} \backslash \alpha_{i} \\ 1 & \text { if } j=\alpha_{i-1}, k=\alpha_{i}\end{cases}
$$

The transition probability matrices corresponding to the finite Markov chain $\left\{Y_{i}\right\}_{i=0}^{n}$ will have the form

$$
\boldsymbol{M}_{i}=\left[\begin{array}{c:c}
N_{i} & C_{i} \\
\hdashline \mathbf{0} & 1
\end{array}\right]
$$

where $\boldsymbol{N}_{i}=\left(p_{i}(k \mid j)\right), j \in \Omega_{i-1} \backslash \alpha_{i-1}$ and $k \in \Omega_{i} \backslash \alpha_{i}$, is an $m_{i-1} \times m_{i}$ rectangular matrix, often referred to as the fundamental matrix, $\boldsymbol{C}_{i}=\left(p_{i}\left(\alpha_{i} \mid j\right)\right)$ is an $m_{i-1} \times 1$ column matrix, and $\mathbf{0}=(0, \ldots, 0)$ is a $1 \times m_{i}$ row matrix. It follows from the Chapman-Kolmogorov equation and the structure of the matrices $\boldsymbol{M}_{i}$ that the probability that the Markov chain $\left\{Y_{i}\right\}_{i=0}^{n}$ never touches the absorbing states, $\mathrm{P}\left(Y_{1} \neq \alpha_{1}, \ldots, Y_{n} \neq \alpha_{n} \mid \xi_{0}\right)$, can be obtained via the following lemma.

Lemma 2.1. Given the state spaces $\left\{\Omega_{i}\right\}_{i=0}^{n}$ and the finite Markov chain $\left\{Y_{i}\right\}_{i=0}^{n}$ defined on the state spaces $\left\{\Omega_{i}\right\}_{i=0}^{n}$ with corresponding transition probability matrices defined by (2.2), we have

$$
\mathrm{P}\left(Y_{1} \neq \alpha_{1}, \ldots, Y_{n} \neq \alpha_{n} \mid \xi_{0}\right)=\xi_{0}\left(\prod_{i=1}^{n} \boldsymbol{N}_{i}\right) \mathbf{1}^{\top},
$$

where the $\boldsymbol{N}_{i}$ are given by (2.1) and (2.2).

While the above construction is somewhat simple, the construction of the sequence of state spaces and the Markov chain $\left\{Y_{i}\right\}_{i=0}^{n}$ with absorbing states will play an indispensable role in our method of computing boundary crossing probabilities. 


\section{Boundary crossing probabilities}

\subsection{Brownian motion}

For given $\Delta t$, the Brownian motion $W(t)$ has transition probability density function

$$
f(x, y \mid \Delta t)=\frac{1}{\sqrt{2 \pi \Delta t}} \exp \left(-\frac{1}{2 \Delta t}(y-x)^{2}\right)
$$

for all $x, y \in \mathbb{R}$. Let $a(t)$ and $b(t)$ be lower and upper boundaries that satisfy the following conditions:

(A) $a(t)<b(t)$ are continuous for $t \in[0, T]$, and

(B) $a(0)<0<b(0)$.

Let $h=\max \left(\sup _{0 \leq t \leq T}|a(t)| \sup _{0 \leq t \leq T}|b(t)|\right)$. Since $a(t)$ and $b(t)$ are continuous functions defined on the compact set $[0, T]$, we have $0<h<\infty$. Given a large positive integer $m$, we define $\Delta x=h / m$ and discretize the real line $\mathbb{R}$ as $\mathbb{R}_{m}=\{k \Delta x: k=0, \pm 1, \ldots\}$. We also discretize the time interval $[0, T]$ into $n=m^{2} T / h^{2}$ subintervals. Without loss of generality, we may assume that $T=1$ and $n=m^{2} / h^{2}$ is always an integer or, if not, we may take $n=\left\lfloor m^{2} / h^{2}\right\rfloor$, the integer part of $m^{2} / h^{2}$. For given $t \in[0,1]$, we then construct a partial sum $\hat{W}_{n}(t)=\sum_{j=1}^{\lfloor n t\rfloor} \hat{X}_{j}$, where $\mathrm{P}\left(\hat{W}_{n}(0)=0\right)=1$ and the $\hat{X}_{j} s$ are discrete, independent and identically distributed (i.i.d.) random variables induced by discretizing the $\mathbb{R}$ and the time interval $[0,1]$, having distribution defined by, for $J=1, \ldots,\lfloor n t\rfloor$,

$$
\mathrm{P}\left(\hat{X}_{J}=k \Delta x\right)= \begin{cases}\frac{C^{-1}}{\sqrt{2 \pi}} \exp \left(-\frac{k^{2}}{2}\right) & \text { if } k \neq 0, \\ \frac{C^{-1}}{\sqrt{2 \pi}} \sum_{\ell \neq 0}\left(\ell^{2}-1\right) \exp \left(-\frac{\ell^{2}}{2}\right) & \text { if } k=0,\end{cases}
$$

where $\sum_{\ell \neq 0}$ stands for $\sum_{\ell=-\infty}^{-1}+\sum_{\ell=1}^{\infty}$ and

$$
C=\frac{1}{\sqrt{2 \pi}} \sum_{\ell \neq 0} \ell^{2} \exp \left(-\frac{\ell^{2}}{2}\right)
$$

is the normalizing constant. Obviously, $\left\{\hat{W}_{n}(t)\right\}$ is a homogeneous Markov chain having transition probabilities

$$
\begin{aligned}
p(k \mid j) & =\mathrm{P}\left(\hat{W}_{n}(t+\Delta t)=k \Delta x \mid \hat{W}_{n}(t)=j \Delta x\right) \\
& = \begin{cases}\frac{C^{-1}}{\sqrt{2 \pi}} \exp \left(-\frac{(k-j)^{2}}{2}\right) & \text { if } k-j \neq 0, \\
\frac{C^{-1}}{\sqrt{2 \pi}} \sum_{\ell \neq 0}\left(\ell^{2}-1\right) \exp \left(-\frac{\ell^{2}}{2}\right) & \text { if } k-j=0 .\end{cases}
\end{aligned}
$$

Note that the discrete probability functions defined by (3.1) have two important characters: (i) they preserve the basic relationship that the variance of $\hat{X}_{j}$ equals $\Delta t=\Delta x^{2}$, and (ii) the transition probabilities depend only on the difference $k-j$. Next we establish that $\hat{W}_{n}(t)$ converges to a Brownian motion $W(t)$ in distribution. 
Theorem 3.1. Given $t \in[0,1]$ and $\Delta t=\Delta x^{2}\left(n=m^{2} / h^{2}\right)$, we have

$$
\hat{W}_{n}(t) \stackrel{\mathrm{D}}{\rightarrow} W(t) \quad \text { as } m \rightarrow \infty,
$$

where $\stackrel{\mathrm{D}}{\rightarrow}$ ' stands for convergence in distribution.

Proof. Note that, for large $m$, the characteristic function of $\hat{X}_{1}$ has the form

$$
\begin{aligned}
\mathrm{E}\left[\mathrm{e}^{i s \hat{X}_{1}}\right] & =\sum_{k \neq 0} \frac{C^{-1}}{\sqrt{2 \pi}}\left(k^{2}-1\right) \mathrm{e}^{-k^{2} / 2}+\sum_{k \neq 0} \mathrm{e}^{i s k \Delta x} \frac{C^{-1}}{\sqrt{2 \pi}} \mathrm{e}^{-k^{2} / 2} \\
& =\frac{C^{-1}}{\sqrt{2 \pi}} \sum_{k \neq 0}\left(k^{2}-1\right) \mathrm{e}^{-k^{2} / 2}+\frac{C^{-1}}{\sqrt{2 \pi}} \sum_{k=1}^{\infty} \mathrm{e}^{-k^{2} / 2}\left(\mathrm{e}^{i s k \Delta x}+\mathrm{e}^{-i s k \Delta x}\right) \\
& =\frac{C^{-1}}{\sqrt{2 \pi}} \sum_{k \neq 0}\left(k^{2}-1\right) \mathrm{e}^{-k^{2} / 2}+\frac{C^{-1}}{\sqrt{2 \pi}} \sum_{k=1}^{\infty} \mathrm{e}^{-k^{2} / 2} 2 \cos (s k \Delta x) \\
& =\frac{C^{-1}}{\sqrt{2 \pi}} \sum_{k \neq 0}\left(k^{2}-1\right) \mathrm{e}^{-k^{2} / 2}+\frac{C^{-1}}{\sqrt{2 \pi}} \sum_{k \neq 0} \mathrm{e}^{-k^{2} / 2}\left(1-\frac{(s k \Delta x)^{2}}{2}+\mathcal{O}\left(\Delta x^{4}\right)\right) \\
& =1-\frac{s^{2} \Delta x^{2}}{2} \frac{C^{-1}}{\sqrt{2 \pi}} \sum_{k \neq 0} k^{2} \mathrm{e}^{-k^{2} / 2}+\mathcal{O}\left(\Delta x^{4}\right) \\
& =1-\frac{s^{2} h^{2}}{2 m^{2}}+\mathcal{O}\left(\frac{1}{m^{4}}\right) .
\end{aligned}
$$

Hence, we have

$$
\varphi_{\hat{W}_{n}(t)}(s)=\left(1-\frac{s^{2} h^{2}}{2 m^{2}}+\mathcal{O}\left(\frac{1}{m^{4}}\right)\right)^{m^{2} t / h^{2}} \rightarrow \exp \left(-\frac{t s^{2}}{2}\right) \quad \text { as } m \rightarrow \infty .
$$

Let $S(t)$ denote a random variable with distribution function to which $\hat{W}_{n}(t)$ converges in distribution. For $\varepsilon>0$, define the event $E_{n}=\{\omega:|S(1 / n)|>\varepsilon\}$. We have $\sum_{n=1}^{\infty} \mathrm{P}\left(E_{n}\right)<\infty$; therefore, by the Borel-Cantelli lemma, $S(t)$ is continuous at $t=0$ almost surely. Obviously, $S(t)$ satisfies the independent increment property; hence, the process $\{S(t), t \in[0,1]\}$ is a Brownian motion owing to its definition.

Remark 3.1. It is worth mentioning that the construction of the probability function in (3.1) which preserves the variance is not unique, for example, we may define the discrete distribution by

$$
\mathrm{P}\left(\hat{X}_{i}=k \Delta x\right)= \begin{cases}\frac{C^{-1}}{k^{p} \sqrt{2 \pi}} \exp \left(-\frac{k^{2}}{2}\right) & \text { if } k \neq 0, \\ \frac{C^{-1}}{\sqrt{2 \pi}} \sum_{\ell \neq 0}\left(\frac{1}{\ell^{p-2}}-\frac{1}{\ell^{p}}\right) \exp \left(-\frac{\ell^{2}}{2}\right) & \text { if } k=0,\end{cases}
$$

where $C=(1 / \sqrt{2 \pi}) \sum_{\ell \neq 0}\left(1 / \ell^{p-2}\right) \exp \left(-\ell^{2} / 2\right)$ and $p$ is even. As $p \rightarrow \infty$, the Markov chain $\left\{\hat{W}_{n}(t: p)\right\}$ induced by $(3.3)$ reduces to a simple random walk moving one step in either the right or left direction with equal probability. For $p=0$, it reduces to (3.1) and $\hat{W}_{n}(t)=\hat{W}_{n}(t: 0)$. We will return to study more about the Markov chain $\hat{W}_{n}(t: p)$ generated by this family of discrete distributions in Section 4. 
In the sequel, we define a nonhomogeneous Markov chain with absorbing states induced by the homogeneous Markov chain $\left\{\hat{W}_{n}(t)\right\}$ and boundaries $a(t)$ and $b(t)$. Let $t_{i}=i \Delta t$, and define $a_{i}=\left\lfloor a\left(t_{i}\right) / \Delta x\right\rfloor$ and $b_{i}=\left\lfloor b\left(t_{i}\right) / \Delta x\right\rfloor$. Then the induced boundaries for $\hat{W}_{n}\left(t_{i}\right)$ are $a^{*}(i / n)=a_{i} \Delta x$ and $b^{*}(i / n)=b_{i} \Delta x$ for $i=1,2, \ldots, n$. We define a finite Markov chain $\left\{Y_{i}(m)\right\}_{i=0}^{n}$ on the state spaces

$$
\Omega_{i}=\left\{j: a_{i}<j<b_{i}\right\} \cup\left\{\alpha_{i}\right\}, \quad i=1,2, \ldots, n,
$$

by collapsing the values of $\hat{W}_{n}\left(t_{i}\right)$ greater than $\left(b_{i}-1\right) \Delta x$ or smaller than $\left(a_{i}+1\right) \Delta x$ into an absorbing $\alpha_{i}$. Then $\left\{Y_{i}(m)\right\}_{i=0}^{n}$ is a nonhomogeneous Markov chain with absorbing states $\left\{\alpha_{i}\right\}$ and transition probabilities given by

$$
\mathrm{P}\left(Y_{i}(m)=k \mid Y_{i-1}(m)=j\right)= \begin{cases}p(k \mid j) & \text { if } j \in \Omega_{i-1} \backslash \alpha_{i-1}, k \in \Omega_{i} \backslash \alpha_{i}, \\ p_{i}\left(\alpha_{i} \mid j\right) & \text { if } j \in \Omega_{i-1} \backslash \alpha_{i-1} \\ 1 & \text { if } j=\alpha_{i-1}, k=\alpha_{i}, \\ 0 & \text { if } j=\alpha_{i-1}, k \in \Omega_{i} \backslash \alpha_{i},\end{cases}
$$

where $p(k \mid j)$ is given by (3.2) and

$$
p_{i}\left(\alpha_{i} \mid j\right)=1-\sum_{k=a_{i}+1}^{b_{i}-1} p(k \mid j)
$$

for all $j \in \Omega_{i-1} \backslash \alpha_{i-1}, \Omega_{0}=\{0\}$, and $\mathrm{P}\left(Y_{0}(m)=0\right) \equiv 1$. All of the transition probability matrices of the Markov chain $\left\{Y_{i}(m)\right\}_{i=0}^{n}$ have the form

$$
\boldsymbol{M}_{i}=\left[\begin{array}{c:c:c}
p(k \mid j) & p_{i}\left(\alpha_{i} \mid j\right) \\
\hdashline \mathbf{0} & 1
\end{array}\right]=\left[\begin{array}{c:c}
\boldsymbol{N}_{i} & \boldsymbol{C}_{i} \\
\hdashline \mathbf{0} & 1
\end{array}\right], \quad i=1,2, \ldots, n,
$$

where the fundamental matrices $N_{i}$ are rectangular of size $\left(b_{i-1}-a_{i-1}-1\right) \times\left(b_{i}-a_{i}-1\right)$. It follows from Lemma 2.1 that the probability that the Markov chain $\left\{Y_{i}(m)\right\}_{i=0}^{n}$ never enters the absorbing states $\alpha_{i}$ is given by the following lemma (see [10]).

Lemma 3.1. Given $m$ and $n=\left\lfloor m^{2} / h^{2}\right\rfloor$, we have

$$
\mathrm{P}\left(Y_{1}(m) \neq \alpha_{1}, \ldots, Y_{n}(m) \neq \alpha_{n} \mid Y_{0}(m)=0\right)=\xi_{0}\left(\prod_{i=1}^{\left\lfloor m^{2} / h^{2}\right\rfloor} \boldsymbol{N}_{i}\right) \mathbf{1}^{\top},
$$

where the $\boldsymbol{N}_{i}, i=1, \ldots, n$, are defined by (3.5) and (3.6), and $\mathbf{1}=(1, \ldots, 1)$ is a row vector of size $\left(b_{n}-a_{n}-1\right)$.

In view of our constructions and Lemma 3.1, we have the following result.

Theorem 3.2. Let $a(t)$ and $b(t)$ be two continuous functions satisfying conditions $(A)$ and $(B)$, and let $W(t)$ be a standard Brownian motion. Then

$$
\mathrm{P}(W(t) \leq a(t) \text { or } W(t) \geq b(t) \text { for some } t \in[0,1])=1-\lim _{m \rightarrow \infty} \xi_{0}\left(\prod_{i=1}^{\left\lfloor m^{2} / h^{2}\right\rfloor} \boldsymbol{N}_{i}\right) \mathbf{1}^{\top} .
$$


Proof. We can rewrite the boundary crossing probability as

$$
\begin{aligned}
\mathrm{P}(a(t) & <W(t)<b(t), 0 \leq t \leq 1) \\
= & \mathrm{P}\left(0<\inf _{0 \leq t \leq 1}(W(t)-a(t)) \text { and } \sup _{0 \leq t \leq 1}(W(t)-b(t))<0\right) .
\end{aligned}
$$

As the time interval $[0,1]$ is divided into $n$ equal subintervals, the boundaries, $a(t)$ and $b(t)$, are also divided into $n$ segments. Then the induced sequences of step functions $a_{n}(t)=a^{*}(\lfloor n t\rfloor / n)$ and $b_{n}(t)=b^{*}(\lfloor n t\rfloor / n)$ uniformly converge to $a(t)$ and $b(t)$, respectively, on the compact set $[0,1]$. Since $a_{n}(t) \rightarrow a(t)$ and $b_{n}(t) \rightarrow b(t)$, by Slutsky's theorem, $\hat{W}_{n}(t)-a_{n}(t)$ and $\hat{W}_{n}(t)-b_{n}(t)$ converge in distribution to $W(t)-a(t)$ and $W(t)-b(t)$, respectively. Also, $h_{1}(x)=\left(\sup _{t} x(t), \inf _{t} x(t)\right)$ is a continuous function. As $m \rightarrow \infty($ or $n \rightarrow \infty)$, the following holds (see [2, p. 77]):

$$
\begin{gathered}
\left(\min _{0 \leq i \leq n}\left(\hat{W}_{n}\left(t_{i}\right)-a^{*}\left(\frac{i}{n}\right)\right), \max _{0 \leq i \leq n}\left(\hat{W}_{n}\left(t_{i}\right)-b^{*}\left(\frac{i}{n}\right)\right)\right) \\
\stackrel{\mathrm{D}}{\rightarrow}\left(\inf _{0 \leq t \leq 1}(W(t)-a(t)), \sup _{0 \leq t \leq 1}(W(t)-b(t))\right) .
\end{gathered}
$$

Hence, the boundary crossing probability for Brownian motion can be approximated via the discrete random walks induced by direct discretization of Brownian motion and calculated using the finite Markov chain imbedding technique as follows:

$$
\begin{aligned}
\mathrm{P}(a(t) & <W(t)<b(t), 0 \leq t \leq 1) \\
= & \mathrm{P}\left(0<\inf _{0 \leq t \leq 1}(W(t)-a(t)) \text { and } \sup _{0 \leq t \leq 1}(W(t)-b(t))<0\right) \\
= & \lim _{m \rightarrow \infty} \mathrm{P}\left(\min _{0 \leq i \leq n}\left(\hat{W}_{n}\left(t_{i}\right)-a^{*}\left(\frac{i}{n}\right)\right)>0 \text { and } \max _{0 \leq i \leq n}\left(\hat{W}_{n}\left(t_{i}\right)-b^{*}\left(\frac{i}{n}\right)\right)<0\right) \\
= & \lim _{m \rightarrow \infty} \mathrm{P}\left(a^{*}\left(\frac{i}{n}\right)<\hat{W}_{n}\left(t_{i}\right)<b^{*}\left(\frac{i}{n}\right) \text { for all } 0 \leq i \leq n\right) \\
= & \lim _{m \rightarrow \infty} \mathrm{P}\left(Y_{1}(m) \neq \alpha_{1}, \ldots, Y_{n}(m) \neq \alpha_{n}\right) \\
= & \lim _{m \rightarrow \infty} \xi_{0}\left(\prod_{i=1}^{\left\lfloor m^{2} / h^{2}\right\rfloor} \boldsymbol{N}_{i}\right) \mathbf{1}^{\top} .
\end{aligned}
$$

This completes the proof.

Remark 3.2. For the one-sided BCP, we simply let $a(t)=-H$ and $H \rightarrow \infty$ (or $b(t)=$ $H$ and $H \rightarrow \infty)$ in our computation. In this case, we use $h=\max \left(H, \sup _{0 \leq t \leq 1} b(t)\right)$ (or $\left.h=\max \left(H, \sup _{0 \leq t \leq 1}|a(t)|\right)\right)$.

\subsection{Diffusion processes}

In this section we extend the method to a class of diffusion processes which can be transformed into functions of a Brownian motion. Two such examples are the Ornstein-Uhlenbeck (OU) process and the Brownian bridge.

An Itô diffusion process is a solution to the stochastic differential equation

$$
\mathrm{d} X(t)=b(t, X(t)) \mathrm{d} t+\sigma(t, X(t)) \mathrm{d} W(t),
$$

where the drift $b(t, x):[0,1] \times \mathbb{R} \rightarrow \mathbb{R}$ and diffusion coefficient $\sigma(t, x):[0,1] \times \mathbb{R} \rightarrow \mathbb{R}$ are 
measurable functions, and $W(t)$ is the standard Brownian motion. The solution of the stochastic differential equation exists uniquely under the growth and Lipschitz conditions (see [12]).

A well-known method for solving the boundary crossing problem for diffusion processes is to express them as functions of a Brownian motion, and the boundary crossing probability for diffusion processes is equivalent to a boundary crossing probability for the Brownian motion with transformed time interval and boundaries. There are a number of papers in the literature concerning the transformation from diffusion processes to a Brownian motion. The one-to-one transformation of the transition probability density functions between diffusion processes described by Kolmogorov's backward equation was first posed by Kolmogorov [13]. Cherkasov [5] established a class of diffusion processes transformed into Brownian motion through one-to-one transformation of the transition probability density functions. Bluman [3] extended Cherkasov's [5] result into a wider class of diffusion processes. Wang and Pötzelberger [27] found a class of diffusion processes which can be expressed as functionals of Brownian motion. It is also known that any time-homogeneous diffusion process can be transformed into a Brownian motion using random time change and a change of variable (see [12, p. 208]).

Using the following well-known results, we give two examples, the OU process and the Brownian bridge, to show the transformations.

- Itô's formula. Let $X(t)$ be an Itô process, $f(t, x)$ be a twice differentiable function on $[0, \infty) \times \mathbb{R}$, and $Y(t)=f(t, X(t))$. Then we have

$$
\mathrm{d} Y(t)=\frac{\partial f}{\partial t} \mathrm{~d} t+\frac{\partial f}{\partial x} \mathrm{~d} X(t)+\frac{1}{2} \frac{\partial^{2} f}{\partial x^{2}} \sigma(t, X(t))^{2} \mathrm{~d} t .
$$

- Random time change. Let $f(t)$ be a continuous function, and let $X(t)$ be a process governed by

$$
\mathrm{d} X(t)=f(t) \mathrm{d} W(t) .
$$

Then a Brownian motion $\tilde{W}\left(\tau_{t}\right)$ is a weak solution, where $\tau_{t}=\int_{0}^{t} f^{2}(s) \mathrm{d} s$ and $g^{-1}(t)=$ $\tau_{t}$ given $f(t)>0$. If $X(0)=x_{0}$ then $\tilde{W}(t)$ starts at $x_{0}$.

Example 3.1. (OU processes.) Let $X(t)$ denote the OU process satisfying

$$
\mathrm{d} X(t)=-\mu X(t) \mathrm{d} t+\sigma \mathrm{d} W(t), \quad X(0)=0 .
$$

It is well known (see, for example, [27]) that the OU process can also be written as

$$
X(t)=\mathrm{e}^{-\mu t} \tilde{W}\left(\tau_{t}\right),
$$

where $\tilde{W}\left(\tau_{t}\right)$ is a Brownian motion with $\tau_{t}=\sigma^{2}\left(\mathrm{e}^{2 \mu t}-1\right) / 2 \mu$.

Example 3.2. (Brownian bridge.) The Brownian bridge is a solution of

$$
\mathrm{d} X(t)=\frac{c-X(t)}{S-t} \mathrm{~d} t+\mathrm{d} W(t) \quad \text { for } 0 \leq t \leq S .
$$

Then we can compute the boundary crossing probability

$$
\begin{aligned}
\mathrm{P}(a(t)<X(t)<b(t), 0 \leq t \leq 1) & =\mathrm{P}\left(a^{\top}(t)<\tilde{W}(t)<b^{\top}(t), 0 \leq t \leq \tau_{1}\right) \\
& =\lim _{m \rightarrow \infty} \xi_{0}\left(\prod_{i=1}^{\left\lfloor m^{2} \tau_{1} / h^{2}\right\rfloor} N_{i}\right) \mathbf{1}^{\top}
\end{aligned}
$$


where

and

$$
a^{\top}(t)=\frac{a(g(t))}{S-g(t)}-\frac{\left((S-g(t)) x_{0}+g(t) c\right) / S}{S-g(t)}
$$

$$
b^{\top}(t)=\frac{b(g(t))}{S-g(t)}-\frac{\left((S-g(t)) x_{0}+g(t) c\right) / S}{S-g(t)} .
$$

\section{Error bound}

Let $\mathcal{F}=\left\{f_{p}\right.$ : the family of distributions given by (3.3) for $\left.p=0,2,4, \ldots\right\}$. For the proposed approximation $\hat{W}_{n}(t: p)$ induced by (3.3) with $f_{p} \in \mathcal{F}$, it is important to know its error bound and numerical performance. Let

$$
\begin{gathered}
\hat{\mathcal{P}}\left(a\left(\frac{k}{n}\right), b\left(\frac{k}{n}\right)\right):=\mathrm{P}\left(a\left(\frac{k}{n}\right)<\hat{W}_{n}\left(t_{k}: p\right)<b\left(\frac{k}{n}\right), k=1, \ldots, n\right), \\
\mathcal{P}(a(t), b(t)):=\mathrm{P}(a(t)<W(t)<b(t), t \in[0,1]) .
\end{gathered}
$$

We present the following theorem.

Theorem 4.1. Given $f_{p} \in \mathcal{F}$,

(i) $\hat{W}_{n}(t: p) \stackrel{\mathrm{D}}{\rightarrow} W(t)$ as $n \rightarrow \infty$, and

(ii) for the boundaries $a(t)$ and $b(t)$ satisfying conditions $(A),(B)$, and

(C) there exists a constant $K$ such that $|a(t+\varepsilon)-a(t)|<K \varepsilon$ and $|b(t+\varepsilon)-b(t)|<$ $K \varepsilon, \varepsilon>0$ (the Lipschitz condition),

then the error bound is

$$
\left|\mathcal{P}(a(t), b(t))-\hat{\mathcal{P}}\left(a^{*}\left(\frac{k}{n}\right), b^{*}\left(\frac{k}{n}\right)\right)\right|=\mathcal{O}\left(\frac{1}{m}\right) \text { as } n \rightarrow \infty,
$$

where $n=\left\lfloor m^{2} / h^{2}\right\rfloor, a^{*}(k / n)$, and $b^{*}(k / n)$ are the boundaries for $\hat{W}_{n}\left(t_{k}: p\right)$.

The proof of the error bound depends on the results of Nagaev [14], [15] and Borokov and Novikov [4]. We list their results as lemmas, but provide no proofs.

Lemma 4.1. ([14], [15].) Assuming that the boundaries $a(t)$ and $b(t)$ satisfy conditions $(A)$, $(B)$, and $(C)$, then there exists a constant $c_{1}$ such that, for any $p=0,2, \ldots, \infty$,

$$
\left|\mathcal{P}(a(t), b(t))-\hat{\mathcal{P}}\left(a\left(\frac{k}{n}\right), b\left(\frac{k}{n}\right)\right)\right|<\frac{c_{1}}{\sqrt{n}},
$$

where $c_{1}$ is a constant which may depend on $p$.

Lemma 4.2. ([4].) Given small $\delta>0$, there exists a constant $c_{2}$ such that

$$
|\mathcal{P}(a(t)-\delta, b(t)+\delta)-\mathcal{P}(a(t)+\delta, b(t)-\delta)|<c_{2} \delta .
$$

Lemma 4.3. Given small $\delta>0$,

$$
\begin{aligned}
& \mid \hat{\mathcal{P}}(\left.a\left(\frac{k}{n}\right)-\delta, b\left(\frac{k}{n}\right)+\delta\right)-\hat{\mathcal{P}}\left(a^{*}\left(\frac{k}{n}\right), b^{*}\left(\frac{k}{n}\right)\right) \mid \\
& \quad \leq\left|\hat{\mathcal{P}}\left(a\left(\frac{k}{n}\right)-\delta, b\left(\frac{k}{n}\right)+\delta\right)-\hat{\mathcal{P}}\left(a\left(\frac{k}{n}\right)+\delta, b\left(\frac{k}{n}\right)-\delta\right)\right| .
\end{aligned}
$$


Proof. From the definitions of $a^{*}(k / n)$ and $b^{*}(k / n)$, the following two inequalities hold for $k=1,2, \ldots, n$ :

$$
a\left(\frac{k}{n}\right)-\delta<a^{*}\left(\frac{k}{n}\right)<a\left(\frac{k}{n}\right)+\delta \quad \text { and } \quad b\left(\frac{k}{n}\right)-\delta<b^{*}\left(\frac{k}{n}\right)<b\left(\frac{k}{n}\right)+\delta .
$$

The lemma follows immediately from the above two inequalities.

Proof of Theorem 4.1. The proof of part (i) follows along the same lines as the proof of Theorem 3.1 and is thus omitted. Given small $\delta>0$, it follows from the triangle inequality that

$$
\begin{aligned}
\left|\mathcal{P}(a(t), b(t))-\hat{\mathcal{P}}\left(a^{*}\left(\frac{k}{n}\right), b^{*}\left(\frac{k}{n}\right)\right)\right| \\
\leq\left|\mathcal{P}(a(t), b(t))-\hat{\mathcal{P}}\left(a\left(\frac{k}{n}\right)-\delta, b\left(\frac{k}{n}\right)+\delta\right)\right| \\
\quad+\left|\hat{\mathcal{P}}\left(a\left(\frac{k}{n}\right)-\delta, b\left(\frac{k}{n}\right)+\delta\right)-\hat{\mathcal{P}}\left(a^{*}\left(\frac{k}{n}\right), b^{*}\left(\frac{k}{n}\right)\right)\right| \\
=A_{n}+B_{n} .
\end{aligned}
$$

Furthermore, using the triangle inequality, we have

$$
\begin{aligned}
A_{n} \leq & |\mathcal{P}(a(t), b(t))-\mathcal{P}(a(t)-\delta, b(t)+\delta)| \\
& +\left|\mathcal{P}(a(t)-\delta, b(t)+\delta)-\hat{\mathcal{P}}\left(a\left(\frac{k}{n}\right)-\delta, b\left(\frac{k}{n}\right)+\delta\right)\right| \\
= & C_{n}+D_{n} .
\end{aligned}
$$

By Lemma 4.3 we have

$$
\begin{aligned}
B_{n} \leq & \left|\hat{\mathcal{P}}\left(a\left(\frac{k}{n}\right)-\delta, b\left(\frac{k}{n}\right)+\delta\right)-\hat{\mathcal{P}}\left(a\left(\frac{k}{n}\right)+\delta, b\left(\frac{k}{n}\right)-\delta\right)\right| \\
\leq & \left|\hat{\mathcal{P}}\left(a\left(\frac{k}{n}\right)-\delta, b\left(\frac{k}{n}\right)+\delta\right)-\mathcal{P}(a(t)-\delta, b(t)+\delta)\right| \\
& +|\mathcal{P}(a(t)-\delta, b(t)+\delta)-\mathcal{P}(a(t)+\delta, b(t)-\delta)| \\
& +\left|\mathcal{P}(a(t)+\delta, b(t)-\delta)-\hat{\mathcal{P}}\left(a\left(\frac{k}{n}\right)+\delta, b\left(\frac{k}{n}\right)-\delta\right)\right| \\
= & E_{n}+F_{n}+G_{n} .
\end{aligned}
$$

It follows from Lemma 4.1 that the $D_{n}, E_{n}$, and $G_{n}$ terms tend to 0 with order $\mathcal{O}(1 / \sqrt{n})$. By Lemma 4.2, the $C_{n}$ and $F_{n}$ terms tend to 0 with order $\mathcal{O}(\delta)$. Part (ii) is an immediate consequence of taking $\delta=h / m$ and $n=\left\lfloor m^{2} / h^{2}\right\rfloor$. This completes the proof of part (ii).

For $p=\infty$, it is a simple random walk (SRW). The result $\hat{W}_{n}(t: \infty) \stackrel{\mathrm{D}}{\rightarrow} W(t)$ was given in [11] and, therefore, we expect the rate of convergence of $\hat{W}_{n}(t: \infty)$ to be slower than that of $\hat{W}_{n}(t: 0)$. In Table 1 we provide numerical results to illustrate the errors and rates of convergence of the BCPs of $\hat{W}_{n}(t: p)$ for various $p$.

Several things can be seen from the Table 1. (i) The error bound tending to 0 is with order $c_{p} / m$ with unknown constant $c_{p}$. (ii) The constant $c_{p}$ not only depends on $p$ but also on the boundaries. For example, in Table 1(a), for $p=0$ and $m=8000$, the error is $1.581 \times 10^{-4}$, 
TABLE 1: The error (|exact - approximation|) for (a) a one-sided Daniels' boundary $\frac{1}{2}-t \log \left(\frac{1}{4}(1+\right.$ $\left.\sqrt{1+8 \mathrm{e}^{-1 / t}}\right)$ and (b) a two-sided boundary $\pm(1+t)$ under various $p$.

\begin{tabular}{rcccc}
\hline \multicolumn{1}{c}{$p=0$} & $p=2$ & $p=6$ & $p=\infty(\mathrm{SRW})$ \\
\hline \multicolumn{5}{c}{ (a) } \\
\hline 2000 & $6.605 \times 10^{-4}$ & $8.780 \times 10^{-4}$ & $9.725 \times 10^{-4}$ & $9.799 \times 10^{-4}$ \\
4000 & $3.220 \times 10^{-4}$ & $4.306 \times 10^{-4}$ & $4.778 \times 10^{-4}$ & $4.809 \times 10^{-4}$ \\
8000 & $1.581 \times 10^{-4}$ & $2.124 \times 10^{-4}$ & $2.360 \times 10^{-4}$ & $2.376 \times 10^{-4}$ \\
12000 & $1.046 \times 10^{-4}$ & $1.408 \times 10^{-4}$ & $1.565 \times 10^{-4}$ & $1.576 \times 10^{-4}$ \\
\hline \multicolumn{5}{c}{ (b) } \\
\hline 2000 & $1.651 \times 10^{-4}$ & $2.220 \times 10^{-4}$ & $2.467 \times 10^{-4}$ & $2.485 \times 10^{-4}$ \\
4000 & $8.158 \times 10^{-5}$ & $1.101 \times 10^{-4}$ & $1.224 \times 10^{-4}$ & $1.233 \times 10^{-4}$ \\
8000 & $4.035 \times 10^{-5}$ & $5.461 \times 10^{-5}$ & $6.013 \times 10^{-5}$ & $6.124 \times 10^{-5}$ \\
12000 & $2.615 \times 10^{-5}$ & $3.623 \times 10^{-5}$ & $3.762 \times 10^{-5}$ & $4.065 \times 10^{-5}$ \\
\hline
\end{tabular}

but, for $p=\infty$ (SRW), it requires $m=12000$ to have the same error. This phenomenon can also be found in Table $1(\mathrm{~b})$. (iii) The $\hat{W}_{n}(t: 0)$ has the fastest rate of convergence to a Brownian motion $W(t)$ among all $f_{p} \in \mathcal{F}$. Technically speaking, we have not yet determined if $f_{0}$ has the best rate among all possible discretizations.

\section{Numerical results}

Numerical results for the standard Brownian motion, OU processes, and the Brownian bridge are given in this section. Tables $2-4$ give BCPs in the time interval $[0,1]$ for various boundaries for Brownian motion and OU processes. In Figure 1 we plot the BCPs for the Brownian bridge.

TABLE 2: One-sided BCPs for Brownian motion.

\begin{tabular}{ccccc}
\hline \multirow{2}{*}{ Boundary } & \multicolumn{4}{c}{$m$} \\
\cline { 2 - 5 } & 100 & 500 & 1000 & 5000 \\
\hline $\exp (-t)$ & 0.558872 & 0.560512 & 0.560866 & 0.561233 \\
$\sqrt{t+1}$ & 0.193925 & 0.195480 & 0.195682 & 0.195935 \\
$1+t-t^{2}$ & 0.253998 & 0.255644 & 0.255915 & 0.256153 \\
$\sin t+1$ & 0.101419 & 0.102643 & 0.102824 & 0.102975 \\
\hline
\end{tabular}

TABLE 3: Two-sided BCPs for Brownian motion.

\begin{tabular}{ccccc}
\hline \multirow{2}{*}{ Boundary } & \multicolumn{4}{c}{$m$} \\
\cline { 2 - 5 } & 100 & 500 & 1000 & 5000 \\
\hline$\pm \exp (-t)$ & 0.984047 & 0.984366 & 0.984406 & 0.984439 \\
$\pm \sqrt{t+1}$ & 0.389771 & 0.391084 & 0.391259 & 0.391403 \\
$\pm\left(1+t-t^{2}\right)$ & 0.509908 & 0.510977 & 0.511128 & 0.511254 \\
\hline
\end{tabular}


TABLE 4: Two-sided BCPs for OU processes for various $\sigma$ and $\rho$ with $m=5000$.

\begin{tabular}{cccccc}
\hline \multirow{2}{*}{ Boundary } & \multicolumn{2}{c}{$\sigma^{2}=0.5$} & & \multicolumn{2}{c}{$\sigma^{2}=2$} \\
\cline { 2 - 3 } \cline { 5 - 6 } & $\rho=0.5$ & $\rho=2$ & & $\rho=0.5$ & $\rho=2$ \\
\hline$\pm(1+t)$ & 0.006915 & 0.000185 & & 0.445738 & 0.250645 \\
$\pm \exp (-t)$ & 0.855233 & 0.745546 & & 0.999629 & 0.999258 \\
$\pm\left(t^{2}+1\right)$ & 0.029596 & 0.002957 & & 0.603617 & 0.430781 \\
\hline
\end{tabular}

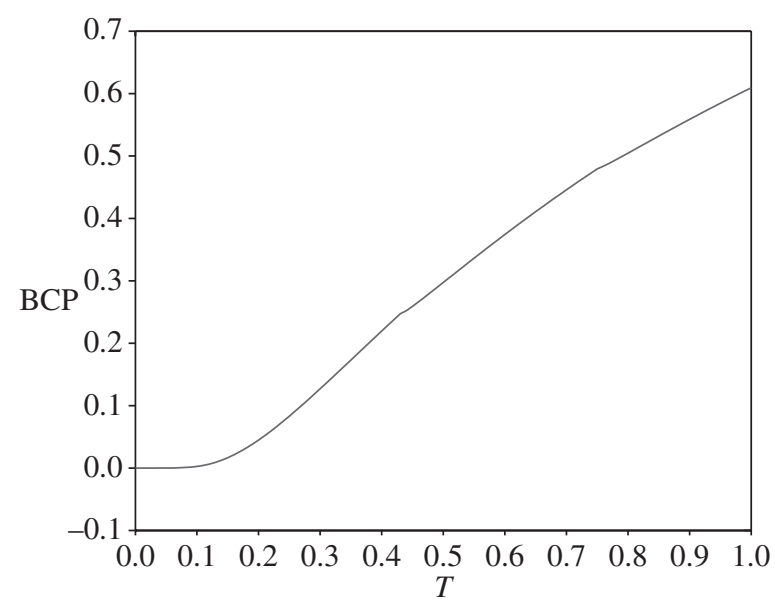

Figure 1: Plot of BCPs for the Brownian bridge with $T \in[0,1], x_{0}=c=0, S=10$, and $\pm \exp (t)$ boundaries.

\section{Summary and discussion}

In this paper we provided a new way to calculate the boundary crossing probabilities for Brownian motion and certain diffusion processes with continuous boundaries, which exploits the advantages of two well-known techniques, the finite Markov chain imbedding technique and the invariance principle. The result involves the multiplication of transition matrices, which is easy to manipulate for computation, even for large $m$. Our method is also flexible in such a way that not only can we compute the BCPs, but we can also calculate the joint distribution of the first passage time and the location of the process (i.e. the probability that the Brownian motion does not cross the boundaries and stays in a certain region of interest at time $T$ ), simply by replacing the column vector $\mathbf{1}^{\top}$ in (3.7) by a vector with $1 \mathrm{~s}$ in the positions of states associated with the region and 0 s elsewhere. The latter probability might be needed in finance problems such as pricing corporate debt.

A well-known result is that the BCP of Brownian motion is 0.479749 with boundary $\frac{1}{2}-$ $t \log \left(\frac{1}{4}\left(1+\sqrt{1+8 \mathrm{e}^{-1 / t}}\right)\right.$ ) (see [6]). By our method, we obtained a BCP of 0.479737 with $m=50000$. For a linear boundary $(1+t)$, our BCP was 0.09041797 with $m=50000$, which is close to the exact BCP of 0.09041777 computed by Robbins and Siegmund [18]. For a twosided boundary $\pm(1+t)$, Anderson [1] obtained a BCP of 0.180812 , and we obtained a BCP of 0.180803 with $m=20000$. Clearly, higher accuracy requires large $m$. The computational CPU times for computing BCPs with $m \leq 5000$ are negligible. 
Another advantage of our method is that it can be extended to compute the BCPs for certain classes of Markov processes. With some proper modifications to the probability functions in (3.1), the method could also be extended to compute the BCPs for two- or higher-dimensional Brownian motion.

\section{Acknowledgements}

We would like to thank the anonymous referee for carefully reading the manuscript and for helpful suggestions. We also would like to thank Dr L. Wang and Dr B. C. Johnson for their comments.

\section{References}

[1] Anderson, T. W. (1960). A modification of the sequential probability ratio test to reduce the sample size. Ann. Math. Statist. 31, 165-197.

[2] Billingsley, P. (1968). Convergence of Probability Measures. John Wiley, New York.

[3] Bluman, G. W. (1980). On the transformation of diffusion processes into the Wiener process. SIAM J. Appl. Math. 39, 238-247.

[4] Borovkov, K. AND Novikov, A. (2005). Explicit bounds for approximation rates of boundary crossing probabilities for the Wiener process. J. Appl. Prob. 42, 82-92.

[5] Cherkasov, I. D. (1957). On the transformation of the diffusion process to a Wiener process. Theory Prob. Appl. 2, 373-377.

[6] Daniels, H. E. (1969). The minimum of stationary Markov process superimposed on a $U$-shaped trend. J. Appl. Prob. 6, 399-408.

[7] Daniels, H. E. (1996). Approximating the first crossing-time density for a curved boundary. Bernoulli 2, 133143.

[8] Durbin, J. (1971). Boundary-crossing probabilities for the Brownian motion and Poisson processes and techniques for computing the power of the Kolmogorov-Smirnov test. J. Appl. Prob. 8, 431-453.

[9] Durbin, J. (1992). The first-passage density of the Brownian motion process to a curved boundary. J. Appl. Prob. 29, 291-304.

[10] Fu, J. C. And Lou, W. Y. W. (2003). Distribution Theory of Runs and Patterns and Its Applications. World Scientific, River Edge, NJ.

[11] KAC, M. (1947). Random walk and the theory of Brownian motion. Amer. Math. Monthly 54, 369-391.

[12] Klebaner, F. C. (2005). Introduction to Stochastic Calculus with Applications. Imperial College, London.

[13] Kolmogorov, A. N. (1931). Über die analytischen methoden in der Wahrscheinlichkeitsrechnung. Math. Ann. 104, 415-458.

[14] Nagaev, S. V. (1970). On the speed of convergence in a boundary value problem. I. Theory Prob. Appl. 15, 163-186.

[15] Nagaev, S. V. (1970). On the speed of convergence in a boundary value problem. II. Theory Prob. Appl. 15, 403-429.

[16] Novikov, A., Frishling, V. And Kordzakhia, N. (1999). Approximations of boundary crossing probabilities for a Brownian motion. J. Appl. Prob. 36, 1019-1030.

[17] Pötzelberger, K. and Wang, L. (2001). Boundary crossing probability for Brownian Motion. J. Appl. Prob. 38, 152-164.

[18] Robbins, H. And Siegmund, D. (1970). Boundary crossing probabilities for Wiener process and sample sums. Ann. Math. Statist. 41, 1410-1429.

[19] Roberts, G. O. and Shortland, C. F. (1997). Pricing barrier options with time-dependent coefficients. Math. Finance 7, 83-93.

[20] Ricciardi, L. M. and Sacerdote, L. (1979). The Ornstein-Uhlenbeck process as a model of neuronal activity. Biol. Cybernet. 35, 1-9.

[21] Ricciardi, L. M., Di Crescenzo, A., Giorno, V. and Nobile, A. G. (1999). An outline of theoretical and algorithmic approaches to first passage time problems with applications to biological modeling. Math. Japon. 50, 247-322.

[22] Sacerdote, L. and Tomassetti, F. (1996). On the evaluations and asymptotic approximations of first-passagetime probabilities. Adv. Appl. Prob. 28, 270-284.

[23] Scheike, T. H. (1992). A boundary-crossing result for Brownian motion. J. Appl. Prob. 29, 448-453.

[24] Siegmund, D. (1986). Boundary crossing probabilities and statistical applications. Ann. Statist. 14, 361-404. 
[25] Strassen, V. (1967). Almost sure behavior of sums of independent random variables and martingales. In Proc. 5th Berkeley Symp. Math. Statist. and Prob., Vol. II, University of California Press, Berkeley, CA, pp. 315-343

[26] Wang, L. ANd Pötzelberger, K. (1997). Boundary crossing probability for Brownian Motion and general boundaries. J. Appl. Prob. 34, 54-65.

[27] Wang, L. and Pötzelberger, K. (2007). Crossing probabilities for diffusion processes with piecewise continuous boundaries. Methodology Comput. Appl. Prob. 9, 21-40. 